Research Article

\title{
Association of Angiopoietin Dysregulation in Placental Malaria with Adverse Birth Outcomes
}

\author{
Puspendra P. Singh, ${ }^{1,2}$ Sneha Bhandari, ${ }^{1}$ Ravendra K. Sharma, ${ }^{1}$ Neeru Singh, ${ }^{1 \dagger}$ \\ and Praveen K. Bharti $\oplus^{1}$ \\ ${ }^{1}$ National Institute of Research in Tribal Health (NIRTH), 482003, Jabalpur, Madhya Pradesh, India \\ ${ }^{2}$ Department of Biological Science, University of Notre Dame, South Bend IN-46556, USA \\ ${ }^{\dagger}$ Deceased \\ Correspondence should be addressed to Praveen K. Bharti; saprapbs@yahoo.co.in
}

Received 16 May 2019; Revised 2 December 2019; Accepted 23 December 2019; Published 13 January 2020

Academic Editor: Michele Malaguarnera

Copyright (c) 2020 Pushpendra P. Singh et al. This is an open access article distributed under the Creative Commons Attribution License, which permits unrestricted use, distribution, and reproduction in any medium, provided the original work is properly cited.

\begin{abstract}
Malaria in pregnancy causes adverse birth outcomes due to sequestration of Plasmodium falciparum-infected erythrocytes in the placenta. Angiopoietins are critical regulators of vascular development and formation of placental villous vasculature. Angiopoietin-1 and Angiopoietin-2 concentrations were measured in peripheral and placental plasma samples from 70 malariainfected and 216 control women using commercially available DuoSet ELISA development kit. Angiopoietins increased in placental plasma (ANG1-5833.5 pg/ml and ANG2-9580.6 pg/ml) as compared to peripheral plasma (ANG1-2293.1 pg/ml and ANG2-1198.9 pg/ml, $p<0.0001)$. The concentration of placental and peripheral ANG1 $(6099.23 \mathrm{pg} / \mathrm{ml}$ and $2320.5 \mathrm{pg} / \mathrm{ml})$ was significantly lower $(5013.5 \mathrm{pg} / \mathrm{ml}, 2208.5 \mathrm{pg} / \mathrm{ml})$, and ANG2 $(9553.3 \mathrm{pg} / \mathrm{ml}, 1180.92 \mathrm{pg} / \mathrm{ml})$ was significantly higher $(9664.6 \mathrm{pg} / \mathrm{ml}, 1254.4 \mathrm{pg} / \mathrm{ml})$ in malaria-positive cases as compared to malaria-negative $(p<0.0001)$. The association of dysregulated angiopoietins in malaria with adverse birth outcomes showed that the peripheral and placental ANG1 concentration was lower and ANG2 concentration was higher in low-birth-weight baby and stillbirth birth outcome as compared to normal deliveries among malaria-positive group. Therefore, ANG1 and ANG2 could be considered a biomarker for adverse outcome during malaria in pregnancy.
\end{abstract}

\section{Introduction}

Plasmodium falciparum infection can cause significant adverse outcomes to mothers and babies. The severity of these consequences depends upon the transmission intensity of the region as it influences the immunity to malaria. In areas with intense transmission of malaria, low birth weight (LBW) and maternal anemia are the major severe outcomes associated with maternal malaria. On the contrary, in low transmission areas, P. falciparum infection can contribute to spontaneous abortion, preterm delivery, intrauterine growth restriction (IUGR), fetal death, and severe malaria in pregnant women [1-3]. Therefore, studies in different endemic regions are needed to better understand the pathogenic mechanisms and risk factors associated with malaria during pregnancy.
Since $P$. falciparum parasites sequester in large numbers in the placenta, intense efforts have been made to understand the sequestration mechanisms, histological changes, and immunological process in the infected placenta [4]. Despite these efforts, biological mechanisms contributing to lowbirth-weight babies, preterm delivery, and stillbirth (SB) in malaria-infected women are not well understood.

Angiopoietins (ANG1 and ANG2) play a vital role in the vascular development [5]. ANG1 and ANG2 function through a common receptor called Tie-2, and alterations in their ratio will have important consequences for their function [6]. Typically, when ANG1 levels are higher than ANG2, it helps to maintain normal vasculature. When the ANG2 level is higher than ANG1, it leads to vascular development [7]. ANG1 and ANG2 have been found to be expressed in placental syncytiotrophoblasts and cytotrophoblasts which 
are important components of placental villi [8]. Sequestration of $P$. falciparum parasites in the placental intervillous blood can potentially alter the relative levels of Ang1/Ang2 and contribute to their functional imbalance [9, 10]. A study reports that ANG1 and ANG2 levels were altered in $P$. falciparum-infected mothers from Africa in favour of a higher ANG2/ANG1 ratio. It was also demonstrated that this dysregulation was associated with low birth weight in malariainfected women [11]. Therefore, it is important to understand the reproducibility of these findings in Asia where the epidemiology of malaria and host immunity is distinct from a typical African malaria setting. We hypothesized that $P$. falciparum malaria infection in pregnant women in an Indian setting may lead to dysregulation of angiopoietins and this may be associated with low birth weight and stillbirth. Therefore, we investigated the role of Ang1 and Ang2 in influencing the adverse outcomes associated with $P$. falciparum infection in pregnant women residing in a low transmission setting in India.

\section{Materials and Methods}

2.1. Study Site and Sampling Method. This study has been carried out during July-2010 to Dec-2012 at the delivery unit at Civil Hospital Maihar, which is a secondary health facility and caters to health needs of rural, semiurban, and ethnic tribal population. Pregnant women who presented at the delivery unit were screened for malaria by microscopic examination of peripheral blood smears. This study had been approved by Institutional Ethics Committee. The women whose peripheral and placental samples were malaria-negative/positive were considered as placental malaria-negative (PM-)/placental malaria-positive $(\mathrm{PM}+)$ group, respectively. The numbers of samples for PM- and $\mathrm{PM}+$ groups were chosen on the premise of roughly the same percentage of low-birth-weight baby (LBW), stillbirth (SB), and gravidity in each group. Written informed consent was taken from pregnant women at the time of enrolment for the study.

2.2. Sample Collection. At the time of enrollment, socioeconomic and clinical data (fever history, clinical symptoms, hemoglobin, and axillary temperature) were recorded. Peripheral blood smears (thick and thin) were prepared for malaria parasite examination, and about $1 \mathrm{ml}$ of peripheral blood was collected for immunological study. After delivery, the expelled placenta was taken for malaria examination and for intervillous blood (incision method) collection. Plasma was separated from all blood samples by centrifugation at $3000 \mathrm{rpm}$ for 10 minutes and stored in $-80^{\circ} \mathrm{C}$ until used for experiments.

2.3. Birth Outcomes. A baby born with no sign of life at or after 28 weeks of gestation was considered a stillbirth. Live birth was defined as complete expulsion of a live baby, irrespective of the duration of the pregnancy. Babies with birth weight below 2500 grams were considered LBW babies, and other babies were considered to have normal birth weight (NBW). Note that birth weight was not measured for stillbirths.

2.4. Measurement of Angiopoietin Levels in Plasma. The peripheral and placental intervillous blood (IVB) plasma levels of ANG1 and ANG2 were measured using the commercially available DuoSet ELISA development kits from $\mathrm{R} \& \mathrm{D}$ systems, according to the manufacturer's instructions. Plasma samples were diluted $1: 4$ and $1: 2$ in the assay buffer for ANG1 and ANG2, respectively.

2.5. Statistical Analysis. Statistical analysis was performed using STATA. Categorical variables were analysed using the chi-squared test, and continuous variables were analysed using the Student $t$-test and ANOVA test to examine the association of angiopoietins in birth outcome and adverse birth outcomes among malaria-positive and malarianegative cases. ROC curve and area under curve (AUC) were also computed to examine the sensitivity and specificity by ANG1 and ANG2 level to discriminate between PM+ and PM- cases.

\section{Results}

A total of 7873 women were screened, and women over the age of 18 with no chronic disease were asked to participate in the study. By microscopy, 101 women were found to have $P$. falciparum infection in both placental and peripheral blood and out of these, 70 were included in the study as placental malaria-positive cases (PM+), the rest of the 31 women were excluded due to missing data either maternal characteristics or birth outcomes. A subset of 216 women (1:3 ratio) whose peripheral and placental smears were negative were considered as placental malaria negative (PM- control group). As summarized in Table 1, PM+ women had a significantly higher body temperature, lower hemoglobin, and lower baby weight as compared to PM- women. Because the samples of malaria-negative and malaria-positive groups were taken as matched in terms of gravidity and birth outcomes, these variables did not and were not expected to have any significant difference among these groups. Peripheral and placental ANG1 level was significantly lower $(p<0.0001)$ and ANG2 was significantly higher $(p<0.0001)$ in $\mathrm{PM}+$ women as compared to PM- women (Table 1).

3.1. Relationship of Angiopoietins with Birth Outcomes. ANG1 was lower in mothers delivering a LBW baby $(2291.1 \mathrm{pg} / \mathrm{ml})$ and SB $(2231.2 \mathrm{pg} / \mathrm{ml})$ birth outcome as compared to normal birth $(2330.8 \mathrm{pg} / \mathrm{ml})$ while ANG2 level was higher in LBW (1197.9 pg/ml) and SB (1225.8 pg/ml) birth outcome as compared to normal birth $(1184.4 \mathrm{pg} / \mathrm{ml})$ in peripheral plasma. In placental plasma, same pattern were found, i.e., ANG1 was lower in LBW $(5780.9 \mathrm{pg} / \mathrm{ml})$ and SB $(5400.8 \mathrm{pg} / \mathrm{ml})$ birth outcome as compared to normal birth $(6135.6 \mathrm{pg} / \mathrm{ml})$ and ANG2 was higher in LBW $(9584.3 \mathrm{pg} / \mathrm{ml})$ and SB $(9624.8 \mathrm{pg} / \mathrm{ml})$ birth outcome as compared to normal $(9551.4 \mathrm{pg} / \mathrm{ml})$ birth. ANOVA analysis was carried out to test the significant association, and the test revealed that lower concentration of peripheral and placental ANG1 protein $(p<0.0001, p<0.0001$, respectively) and higher 
TABLE 1: Mother's characteristics and birth outcomes by placental malaria status.

\begin{tabular}{|c|c|c|c|}
\hline & $\begin{array}{c}\text { Malaria (-ve) } \\
\quad N=216\end{array}$ & $\begin{array}{c}\text { Malaria (+ve) } \\
\quad N=70\end{array}$ & $p$ value $^{* *}$ \\
\hline \multicolumn{4}{|l|}{ Characteristics of mother } \\
\hline Age $^{\mathrm{a}}$ & $24.1 \pm 3.8$ & $24.7 \pm 4.0$ & 0.27 \\
\hline $\begin{array}{l}\text { Axillary } \\
\text { temperature }\left({ }^{\circ} \mathrm{C}\right)^{\mathrm{a}}\end{array}$ & $36.0 \pm 1.4$ & $36.7 \pm 1.3$ & 0.002 \\
\hline Haemoglobin $(\mathrm{g} / \mathrm{dl})^{\mathrm{a}}$ & $11.2 \pm 2.6$ & $09.6 \pm 2.2$ & $<0.0001$ \\
\hline Anaemia & $89(41 \%)$ & $49(70 \%)$ & $<0.0001$ \\
\hline \multicolumn{4}{|l|}{ Gravidae* } \\
\hline Primgravid & $72(33.3 \%)$ & $25(35.7 \%)$ & \\
\hline Secundigravid & $71(32.9 \%)$ & $22(31.4 \%)$ & \\
\hline Multigravid & $73(33.8 \%)$ & $23(32.9 \%)$ & \\
\hline \multicolumn{4}{|c|}{ Angiopoietin $(\mathrm{pg} / \mathrm{ml})^{\mathrm{a}}$} \\
\hline Peripheral ANG1 & $2320.5 \pm 138.7$ & $2208.5 \pm 101.3$ & $<0.0001$ \\
\hline Peripheral ANG2 & $1180.9 \pm 67.0$ & $1254.4 \pm 66.5$ & $<0.0001$ \\
\hline Placental ANG1 & $6099 \pm 1177.9$ & $5013.6 \pm 831.2$ & $<0.0001$ \\
\hline Placental ANG2 & $9553.3 \pm 119.0$ & $9664.6 \pm 88.2$ & $<0.0001$ \\
\hline \multicolumn{4}{|l|}{ Birth outcomes } \\
\hline Normal* & $83(38.4 \%)$ & $28(40.0 \%)$ & \\
\hline Low birth weight* & $86(39.8 \%)$ & $25(35.7 \%)$ & \\
\hline Stillbirth* & $47(21.8 \%)$ & $17(24.3 \%)$ & \\
\hline $\begin{array}{l}\text { Baby weight } \\
(\mathrm{gm})^{\mathrm{a} \#}\end{array}$ & $2470.4 \pm 382.0$ & $2292.8 \pm 442.8$ & 0.005 \\
\hline
\end{tabular}

${ }^{a}$ Mean $\pm \mathrm{SD}$; ${ }^{*}$ indicates variable used to frequency match PM+ and PMgroups; ${ }^{* *} p$ values reported are Pearson chi-square test for categorical variable and two sample $t$-test for continuous variables; "Birth weight information was collected for 53 babies born from PM+ and 169 babies born from PM- women.

concentration of peripheral and placental ANG2 protein $(p<0.001)$ were significantly associated with SB birth outcome as compared to normal birth outcome except in the case of peripheral ANG2 for mothers with low birth weight (Table 2). The ANOVA analysis also reveals that the ratio of peripheral ANG1 and ANG2 and ratio of placental ANG1 and ANG2 also vary considerably among normal, low birth weight, and stillbirth. Ratios of peripheral ANG1/ANG2 and placental ANG1/ANG2 were significantly higher in LBW and SB birth categories.

3.2. Level of Angiopoietin among Birth Outcome within Malaria Status. We explored the angiopoietin dysregulation and their association with PM and birth outcomes. The peripheral ANG1 level was significantly lower in normal birth (2276.1 $\pm 58.8 v s 2349.2 \pm 139.8)$, low birth weight (2222.9 $\pm 81.6 v s 2310.9 \pm 159.8)$, and stillbirth $(2075.9 \pm 37.3 v s 2287.3 \pm 71.4)$ in the PM+ group compared to the PM- group. Similarly, the placental ANG1 level was significantly lower in the PM+ group compared to the PM- group in all three birth outcomes. But, on contrast, the peripheral ANG2 level was significantly higher in
$\mathrm{PM}+$ compared to PM- in normal birth $(1216.3 \pm 70.0$ $v s 1173.6 \pm 69.7)$, low birth weight $(1258.7 \pm 53.2$ $v s 1180.3 \pm 77.6), \quad$ and stillbirth $\quad(1310.7 \pm 24.2$ $v s 1195.1 \pm 29.6$ ). Similarly, in case of placental plasma values, ANG2 was higher in $\mathrm{PM}+$ group compared to PM- groups in all birth outcomes categories (Table 3). Statistical analysis to test the significant association of angiopoietin dysregulation in birth outcome category among $\mathrm{PM}-$ and PM+ group revealed that the peripheral ANG1 level in the $\mathrm{PM}+$ group was significantly lower in LBW $(2222.9 \pm 81.6 v s 2276.1 \pm 58.8)$ and SB birth $(2075.9 \pm 37.3$ vs2276.1 \pm 58.8 ) outcome as compared to normal, whereas a high level of peripheral ANG2 in PM+ was seen in LBW $(1258.7 \pm 53.2 v s 1216.3 \pm 70.0)$ and $\mathrm{SB}$ birth outcome $(1310.7 \pm 24.2 v s 1216.3 \pm 70.0)$ as compared to normal birth outcomes. The angiopoietin level of placental plasma in the $\mathrm{PM}+$ group also showed the same pattern (i.e., a significant decrease of ANG1 and increase of ANG2) in adverse birth outcome as compared to normal. The ratio of peripheral (AGN2/AGN1) was statistically higher in the PM+ group compared to the PM- group within the birth outcome categories and among $\mathrm{PM}+$ cases in LBW $(0.57 \pm 0.03$ $v s 0.53 \pm 0.03)$ and $\mathrm{SB}(0.63 \pm 0.02 v s 0.53 \pm 0.03)$ compared to normal birth outcome. Similarly, the ratio of placental (AGN2/AGN1) was statistically higher in the $\mathrm{PM}+$ group compared to the PM- group within the birth outcome categories and among $\mathrm{PM}+$ cases in LBW $(1.99 \pm 0.11$ $v s 1.75 \pm 0.28)$ and $\mathrm{SB}(0.2 .34 \pm 0.23 v s 1.75 \pm 0.28)$ compared to normal birth outcome (Table 3 ).

Statistical analysis to test the significant association of angiopoietin dysregulation in birth outcome category among the $\mathrm{PM}$ - and $\mathrm{PM}+$ groups revealed that the peripheral ANG1 level in the $\mathrm{PM}+$ group was significantly lower in LBW $(p<0.02)$ and SB $(p<0.0001)$ birth outcome as compared to normal. A high level of peripheral ANG2 in $\mathrm{PM}+$ was seen in LBW $(p<0.02)$ and SB $(p<0.0001)$ birth outcome as compared to normal birth outcomes. The angiopoietin level of placental plasma in the $\mathrm{PM}+$ group also showed the same pattern (i.e., a significant decrease of ANG1 and increase of ANG2) in adverse birth outcome as compared to normal (Figure 1).

A receiver operating characteristic (ROC) curve was drawn to predict the sensitivity and specificity of ANG1 and ANG2 levels with birth outcome in malaria-positive mothers. The ROC curve and area under the curve show that all variables in peripheral ANG1 and ANG2, placental ANG1 and ANG2, and ratio of peripheral (ANG2/ANG1) and placental (ANG2/ANG1) show a significantly higher area than the hypothetical area under curve 0.5. Area under curve of ratio of ANG2/ANG1 was 0.86 in the case of peripheral and 0.83 in placental malaria-positive mothers in case of LBW. Similarly, in the case of SB ratio, ANG2/ANG1 was 0.98 in peripheral and 0.94 in placental malaria-positive mothers (Figure 2).

\section{Discussion}

Human pregnancy is characterized by angiogenesis, tissue development, and remodeling $[12,13]$. The disturbance in 
TABLE 2: Angiopoietin concentration (pg/ml) among birth outcome category.

\begin{tabular}{lcccccc}
\hline \multirow{2}{*}{ Birth outcomes } & & \multicolumn{2}{c}{ Mean \pm SD } & & Ratio of peripheral \\
& Peripheral ANG1 & Peripheral ANG2 & Placental ANG1 & Placental ANG2 & $\begin{array}{c}\text { Ratio placental } \\
\text { ANG2/ANG1 }\end{array}$ & ANG2/ANG1 \\
\hline Normal (111) & $2330.8 \pm 128.2$ & $1184.4 \pm 71.9$ & $6135.6 \pm 1269.4$ & $9551.4 \pm 131.8$ & $0.510 \pm 0.040$ & $1.624 \pm 0.340$ \\
LBW (111) & $2291.1^{*} \pm 150.2$ & $1197.9 \pm 79.8$ & $5780.9^{*} \pm 1165.6$ & $9584.3^{*} \pm 102.1$ & $0.526^{*} \pm 0.054$ & $1.724^{*} \pm 0.338$ \\
SB (64) & $2231.2^{* *} \pm 113.7$ & $1225.8^{* *} \pm 58.6$ & $5400.8^{* *} \pm 969.0$ & $9624.8^{* *} \pm 123.4$ & $0.552^{* *} \pm 0.052$ & $1.846^{* *} \pm 0.370$ \\
Total (286) & $2293.1 \pm 139.0$ & $1198.9 \pm 73.9$ & $5833.5 \pm 1196.9$ & $9580.6 \pm 121.9$ & $0.525 \pm 0.051$ & $1.713 \pm 0.355$ \\
& 11.183 & 6.663 & 8.221 & 7.783 & 15.549 & 8.422 \\
F-statistics (p) & $p \leq 0.0001$ & $p \leq 0.001$ & $p \leq 0.0001$ & $p \leq 0.001$ & $p \leq 0.0001$ & $p \leq 0.0001$ \\
\hline
\end{tabular}

Note: Level of significance between normal and LBW/SB based on $t$-test, ${ }^{* *} p \leq 0.01,{ }^{*} p \leq 0.05$.

TABLE 3: Angiopoietin concentration $(\mathrm{pg} / \mathrm{ml})$ in birth outcome category with malaria status.

\begin{tabular}{|c|c|c|c|c|c|c|c|}
\hline \multirow[b]{2}{*}{$\begin{array}{l}\text { Birth } \\
\text { outcome }\end{array}$} & \multirow[b]{2}{*}{ Malaria result } & \multicolumn{6}{|c|}{ Mean \pm SD } \\
\hline & & $\begin{array}{l}\text { Peripheral } \\
\text { ANG1 }\end{array}$ & $\begin{array}{l}\text { Peripheral } \\
\text { ANG2 }\end{array}$ & Placental ANG1 & $\begin{array}{c}\text { Placental } \\
\text { ANG2 }\end{array}$ & $\begin{array}{l}\text { Ratio of peripheral } \\
\text { ANG2/ANG1 }\end{array}$ & $\begin{array}{c}\text { Ratio of placental } \\
\text { ANG2/ANG1 }\end{array}$ \\
\hline \multirow{2}{*}{ Normal } & Negative (83) & $2349.2 \pm 139.8$ & $1173.6 \pm 69.7$ & $6307.5 \pm 1335.0$ & $9536.1 \pm 146.1$ & $0.50 \pm 0.04$ & $1.58 \pm 0.35$ \\
\hline & Positive (28) & $2276.1^{* *} \pm 58.8$ & $1216.3^{* *} \pm 70.0$ & $5626.1^{* *} \pm 890.0$ & $9596.7^{* *} \pm 55.4$ & $0.53^{* *} \pm 0.03$ & $1.75^{*} \pm 0.28$ \\
\hline \multirow{2}{*}{ LBW } & Negative (86) & $2310.9 \pm 159.8$ & $1180.3 \pm 77.6$ & $6045.7 \pm 1192.1$ & $9561.5 \pm 103.0$ & $0.51 \pm 0.05$ & $1.65 \pm 0.34$ \\
\hline & Positive (25) & $2222.9^{* *} \pm 81.6$ & $1258.7^{* *} \pm 53.2$ & $4869.9^{* *} \pm 280.9$ & $9662.9^{* *} \pm 43.7$ & $0.57^{* *} \pm 0.03$ & $1.99^{* *} \pm 0.11$ \\
\hline \multirow{2}{*}{ SB } & Negative (47) & $2287.3 \pm 71.4$ & $1195.1 \pm 29.6$ & $5829.4 \pm 723.9$ & $9568.9 \pm 87.3$ & $0.52 \pm 0.02$ & $1.67 \pm 0.22$ \\
\hline & Positive (17) & $2075.9^{* *} \pm 37.3$ & $1310.7^{* *} \pm 24.2$ & $4216.0^{* *} \pm 399.7$ & $9779.2^{* *} \pm 59.3$ & $0.63^{* *} \pm 0.02$ & $2.34^{* *} \pm 0.23$ \\
\hline \multirow{2}{*}{ Total } & Negative (216) & $2320.5 \pm 138.7$ & $1180.9 \pm 67.0$ & $6099.2 \pm 1177.9$ & $9553.3 \pm 119.0$ & $0.51 \pm 0.04$ & $1.63 \pm 0.32$ \\
\hline & Positive (70) & $2208.5^{* *} \pm 101.3$ & $1254.4^{* *} \pm 66.5$ & $5013.6^{* *} \pm 831.2$ & $9664.7^{* *} \pm 88.2$ & $0.57^{* *} \pm 0.05$ & $1.98^{* *} \pm 0.32$ \\
\hline \multicolumn{2}{|c|}{ Grand total (286) } & $2293.1 \pm 139.0$ & $1198.9 \pm 73.9$ & $5833.5 \pm 1196.9$ & $9580.6 \pm 121.9$ & $0.53 \pm 0.05$ & $1.71 \pm 0.35$ \\
\hline
\end{tabular}

Note: Level of significance between negative and positive cases based on $t$-test, ${ }^{* *} p \leq 0.01,{ }^{*} p \leq 0.05$.

the balance of proangiogenic and antiangiogenic factors can lead to impaired placentation, causing major pregnancy complications such as preeclampsia and intrauterine growth restriction which can lead to poor obstetric outcomes [14]. Human parturition has a common pathway characterized by increased uterine contractility, cervical ripening/dilation, and membrane/decidual activation, culminating in membrane rupture [15]. This activation is a coordinated inflammatory phenomenon in normal spontaneous labor during the term delivery $[16,17]$. This hypothesis is also proven in a mouse model as increased expression of angiogenesisrelated genes in mouse uterus was observed in spontaneous labor at term delivery as well as pathologically induced preterm labor [18].

In the present study, the angiopoietin level was significantly elevated in the placental plasma as compared to the peripheral plasma which supports the previous findings that endometrium, deciduas, and placenta are rich sources of angiogenic growth factor [19, 20]. Many studies reviewed by Zygmunt et al. showed that angiopoietins play an important role for successful placentation as well as parturition [21]. Placental vascular development and remodeling are controlled primarily through the highly regulated actions of angiogenic factors from the VEGF and angiopoietin families [12]. ANG1 has a major role in the stabili- zation and maturation of newly formed vessels, whereas ANG2 provides the destabilizing effect necessary to initiate vascular remodeling $[22,23]$. The downregulation of ANG1 and upregulation of ANG2 in P. falciparum-infected cases from the present study are very much similar to previous studies $[11,24]$. The angiogenic factor perturbation has also been reported in preeclampsia and fetal growth restriction associated with inadequate placental vascularization [25]. Normally, the ANG1 level increases and ANG2 level decreases throughout the gestation but alterations in the levels of angiopoietins may cause pathological pregnancies $[12,13,26]$. Some studies related to association of angiopoietins in cerebral malaria (CM) demonstrated that ANG1 act as a good biomarker in differentiating CM from mild malaria among Thai adults, but not in Ugandan children [27]. Yeo et al. reported that ANG2 is a better marker of severe malaria-associated deaths than lactate in Indonesian adults [10]. Among Ugandan children, higher levels of ANG1 were found to be associated with a reduced risk of death [27]. In a recent study conducted with Malawian children, ANG1 levels were significantly downregulated among CM patients with retinopathy compared to those without retinopathy, uncomplicated malaria patients, and those with nonmalarial encephalopathy [28]. Involvement of ANG1 and ANG2 in the pathogenesis of cerebral malaria 

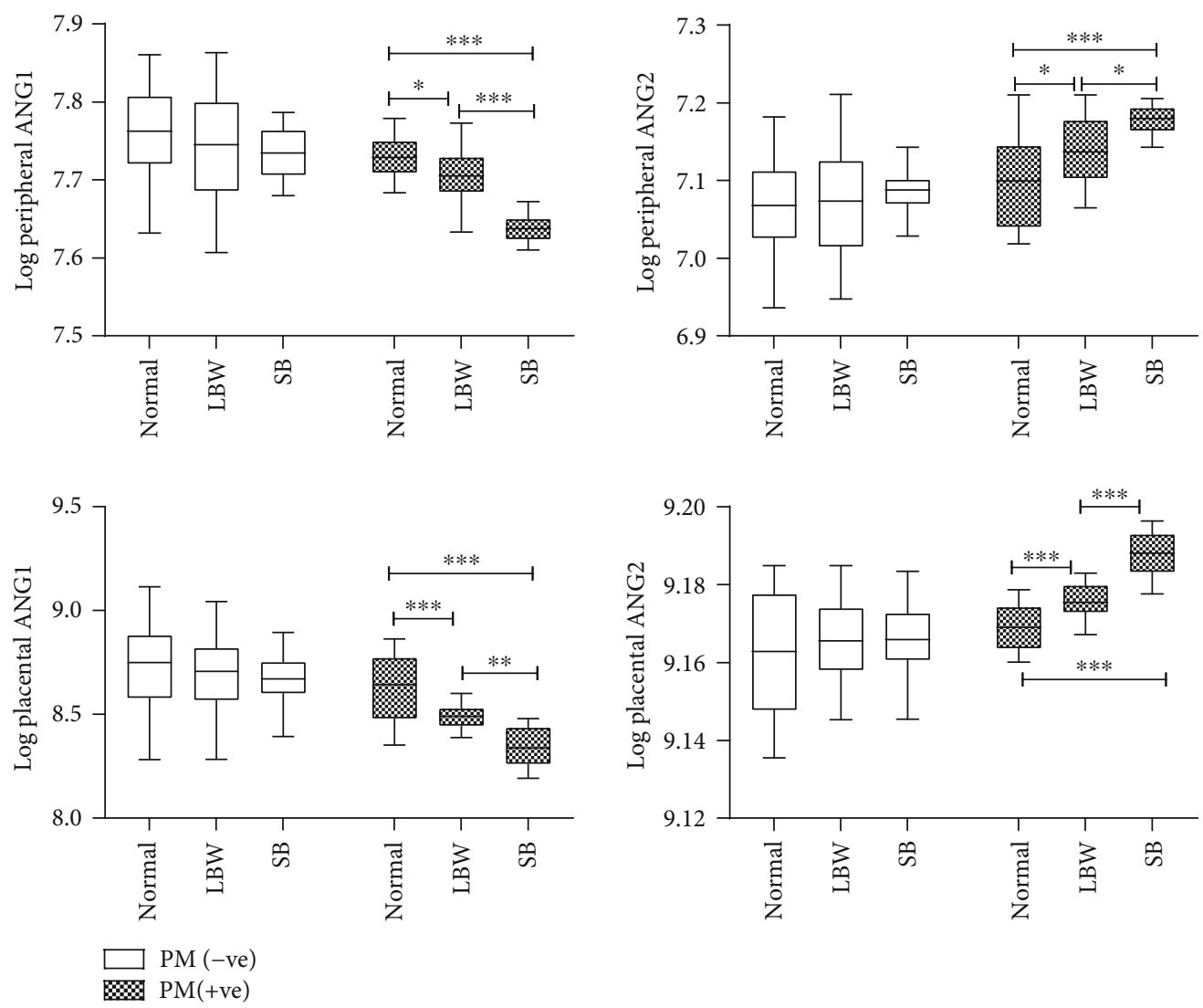

Figure 1: Angiopoietin level in birth outcome category among the PM- and PM+ groups. Box plot shows the median, interquartile range with whiskers denoting the maximum and minimum values. ${ }^{*} p<0.02,{ }^{* *} p<0.002,{ }^{* * *} \mathrm{p}<0.0001$.

was also studied in Central India and revealed that the concentration of ANG1 was lower in the cerebral malaria survivor and nonsurvivor groups as compared to mild malaria and healthy control. ANG2 showed positive association with malaria severity [29].

Consistent with earlier studies, the present study highlights that the altered level of angiopoietin due to malaria (infection/inflammation) may be associated with the pathogenesis of birth outcomes. Placental malaria (PM) is believed to lead to placental insufficiency, a progressive deterioration in placental function and inability to sustain fetal growth, resulting in LBW infants and also increased risk of perinatal mortality [30]. Previous reports are consistent with the hypothesis that PM may influence angiogenesis and vascular development. Histological and ultrasound studies of PM suggest that malaria infection alters the placental vascular structure [31, 32]. Altered levels of angiopoietins, sEng, VEGF, sFlt-1, or C5a have recently been shown in several populations of African women to have with PM at delivery $[11,24,33]$. Although the mechanism of adverse birth outcomes (LBW and stillbirth) is poorly understood, it is hypothesized that these pregnancy outcomes may result of intrauterine growth restriction and/or premature delivery [34]. Both IUGR and spontaneous premature delivery can result from functional placental insufficiency where nutrient supply is inadequate to support fetal growth and continued in utero-development [35].

The mean concentration of ANG1 was decreased, and ANG2 was increased in women with malaria who delivered LBW and stillbirth baby in the current study. These findings are in line with those earlier reports that support that the dysregulation of angiopoietins was associated with complicated pregnancy outcome [36]. Low ANG1 was reported to lead to vessel destabilization and a decrease in the angiogenic sprouting promoting vessel leakage [37] which may be the explanation of adverse birth outcome. Several scenarios have been proposed to account for the serum measurement of angiogenic factors in maternal blood according to early pregnancy state. Involvement of angiogenic factors in miscarriage, abnormal placentation, preeclampsia, and IUGR pregnancies was also shown in various studies [38]. The study of Conroy et al. also suggested that activation of complement system ( $\mathrm{C} 5 \mathrm{a}$ ) due to malaria caused dysregulation of angiogenic factor, i.e., ANG1 was negatively and ANG2 was positively associated with complement activation which was found to be associated with fetal growth restrictions [39].

The finding of this present study is consistent with few published studies which can lead to established ANG1 and ANG2 as biomarker for the severity due to malaria during pregnancy. 

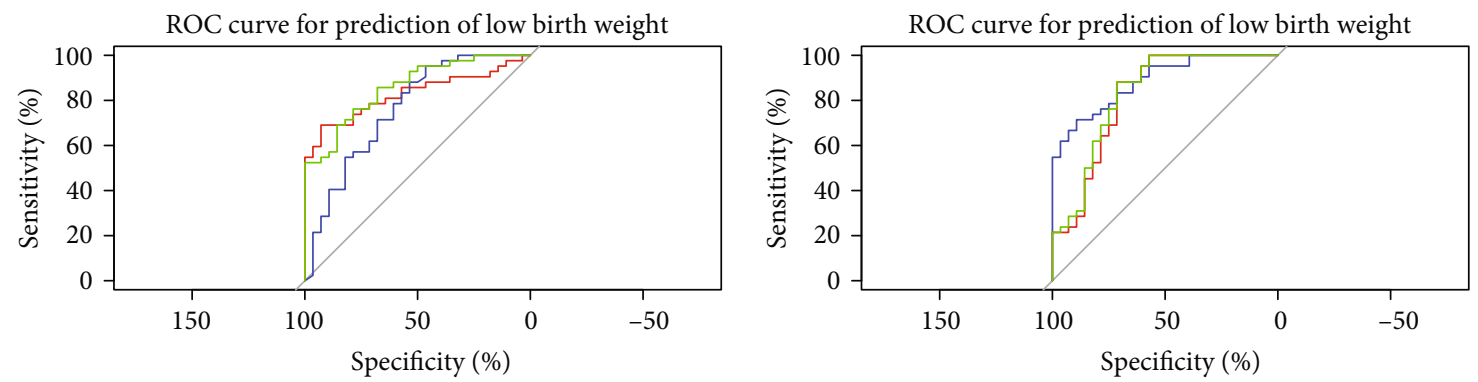

Periperal (malaria +ve)

— ANG1 [AUC: 0.83(0.73-0.92)]

— ANG2 [AUC: 0.76(0.64-0.88)]

— ANG2/ANG1 ratio [AUC: $0.86(0.77-0.94)]$

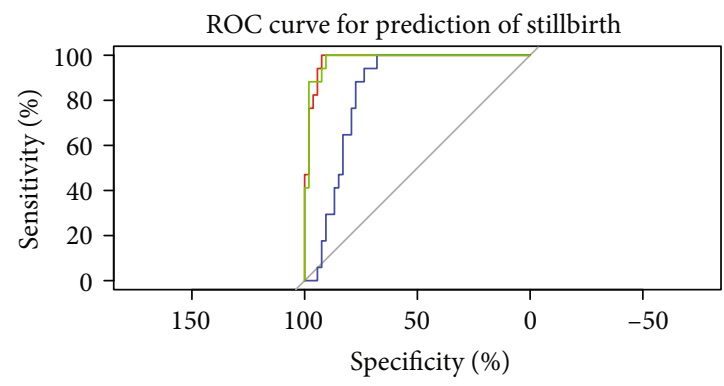

Periperal (malaria $+\mathrm{ve})$

- ANG1 [AUC: 0.98(0.96-1.0)]

— ANG2 [AUC: 0.84(0.75-0.93)]

— ANG2/ANG1 ratio [AUC: 0.98(0.96-1.0)]

Placental (malaria +ve)

— ANG1 [AUC: 0.82(0.70-0.93)]

— ANG2 [AUC: 0.88(0.81-0.96)]

— ANG2/ANG1 ratio [AUC: 0.83(0.72-0.94)]

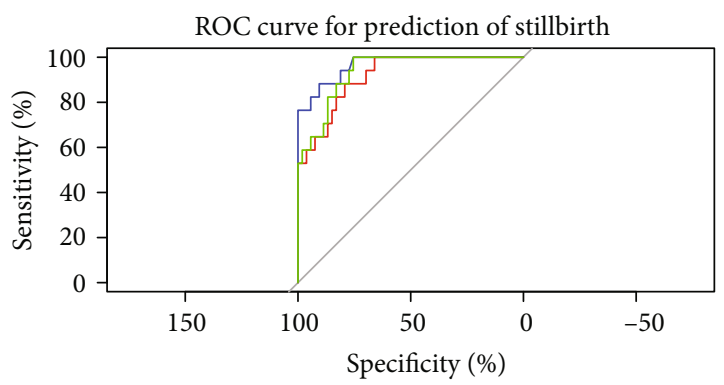

Placental (malaria +ve)

- ANG1 [AUC: 0.92(0.85-0.98)]

— ANG2 [AUC: 0.97(0.93-1.0)]

_ ANG2/ANG1 ratio [AUC: 0.94(0.88-0.99)]

FIGURE 2: ROC curve of peripheral and placental angiopoietin levels in birth outcome category among malaria-positive mothers.

\section{Data Availability}

We have reported all the findings in the manuscript. The patient information sheets are available with the corresponding author.

\section{Ethical Approval}

The study has been approved by Institutional Ethics Committee of ICMR-NIRTH Jabalpur and the manuscript is approved by Institutional Publication Screening Committee.

\section{Disclosure}

The statements made herein are solely the responsibility of the author(s).

\section{Conflicts of Interest}

The authors declare that there was no competing financial interest.

\section{Authors' Contributions}

Praveen Kumar Bharti is involved in conceptualization, data curation, funding acquisition, project administration, resources, supervision, and writing-review and editing. Pushpendra P Singh is involved in conceptualization, data curation, formal analysis, investigation, and writing—original draft. Sneha Bhandari is involved in data curation, investigation, formal analysis, and writing-original draft. Ravendra $\mathrm{K}$ Sharma is involved in data curation, formal analysis, investigation, and writing-original draft, review, and editing. Neeru Singh is involved in conceptualization and resources. All authors read and approved the final manuscript.

\section{Acknowledgments}

This publication was made possible by ICMR- SRF from the Indian Council of Medical Research Fund. We thank all the pregnant women who participated in this study and our technicians/staff nurses for their continuous support. Special thanks to Dr. S. B Awadiya (Gynaecologist, Civil Hospital, Maihar), for her kind support during the sample collection at study site. We regret to inform that the author Dr. Neeru Singh passed away before the publication of the research work. This study was funded by the Indian Council of Medical Research under ICMRSRF fellowship.

\section{References}

[1] C. Luxemburger, F. Ricci, F. Nosten, D. Raimond, S. Bathet, and N. J. White, "The epidemiology of severe malaria in an area of low transmission in Thailand," Transactions of the Royal Society of Tropical Medicine and Hygiene, vol. 91, no. 3, pp. 256-262, 1997. 
[2] R. McGready, S. J. Lee, J. Wiladphaingern et al., "Adverse effects of falciparum and vivax malaria and the safety of antimalarial treatment in early pregnancy: a population-based study," The Lancet Infectious Diseases, vol. 12, no. 5, pp. 388-396, 2012.

[3] J. M. McDermott, J. G. Breman, J. J. Wirima, D. L. Heymann, and R. W. Steketee, "The effect of placental malaria infection on perinatal mortality in rural Malawi," The American Journal of Tropical Medicine and Hygiene, vol. 55, 1_Suppl, pp. 61-65, 1996.

[4] J. N. Bulmer, F. N. Rasheed, N. Francis, L. Morrison, and B. M. Greenwood, "Placental malaria. I. Pathological classification," Histopathology, vol. 22, no. 3, pp. 211-218, 1993.

[5] M. Thomas and H. G. Augustin, "The role of the angiopoietins in vascular morphogenesis," Angiogenesis, vol. 12, no. 2, pp. 125-137, 2009.

[6] P. C. Maisonpierre, C. Suri, P. F. Jones et al., "Angiopoietin-2, a natural antagonist for Tie2 that disrupts in vivo angiogenesis," Science, vol. 277, no. 5322, pp. 55-60, 1997.

[7] D. Hanahan, "Signaling vascular morphogenesis and maintenance," Science, vol. 277, no. 5322, pp. 48-50, 1997.

[8] E. Geva and R. B. Jaffe, "Role of angiopoietins in reproductive tract angiogenesis," Obstetrical \& Gynecological Survey, vol. 55, no. 8, pp. 511-519, 2000.

[9] A. Conroy, L. Serghides, C. Finney et al., "C5a enhances dysregulated inflammatory and angiogenic responses to malaria in vitro: potential implications for placental malaria," PLoS One, vol. 4, no. 3, p. e4953, 2009.

[10] T. W. Yeo, D. A. Lampah, R. Gitawati et al., "Angiopoietin-2 is associated with decreased endothelial nitric oxide and poor clinical outcome in severe falciparum malaria," Proceedings of the National Academy of Sciences, vol. 105, no. 44, pp. 17097-17102, 2008.

[11] K. L. Silver, K. Zhong, R. G. F. Leke, D. W. Taylor, and K. C. Kain, "Dysregulation of angiopoietins is associated with placental malaria and low birth weight," PloS One, vol. 5, no. 3, p. e9481, 2010.

[12] E. Geva, D. G. Ginzinger, C. J. Zaloudek, D. H. Moore, A. Byrne, and R. B. Jaffe, "Human placental vascular development: vasculogenic and angiogenic (branching and nonbranching) transformation is regulated by vascular endothelial growth factor-a, angiopoietin-1, and angiopoietin-2," The Journal of Clinical Endocrinology \& Metabolism, vol. 87, no. 9, pp. 4213-4224, 2002.

[13] E. G. Zhang, S. K. Smith, P. N. Baker, and D. S. CharnockJones, "The regulation and localization of angiopoietin-1, -2 , and their receptor Tie2 in normal and pathologic human placentae," Molecular Medicine, vol. 7, no. 9, pp. 624-635, 2001.

[14] V. Gourvas, E. Dalpa, A. Konstantinidou, N. Vrachnis, D. A. Spandidos, and S. Sifakis, "Angiogenic factors in placentas from pregnancies complicated by fetal growth restriction (review)," Molecular Medicine Reports, vol. 6, no. 1, pp. 23-27, 2012.

[15] R. Romero, J. Espinoza, J. P. Kusanovic et al., “The preterm parturition syndrome," BJOG: An International Journal of Obstetrics \& Gynaecology, vol. 113, pp. 17-42, 2006.

[16] A. J. Thomson, "Leukocytes infiltrate the myometrium during human parturition: further evidence that labour is an inflammatory process," Human Reproduction, vol. 14, no. 1, pp. 229-236, 1999.
[17] A. Young, A. J. Thomson, M. A. Ledingham, F. Jordan, I. A. Greer, and J. E. Norman, "Immunolocalization of proinflammatory cytokines in myometrium, cervix, and fetal membranes during human parturition at term," Biology of Reproduction, vol. 66, no. 2, pp. 445-449, 2002.

[18] R. Haddad, R. Romero, B. R. Gould et al., "Angiogenesis gene expression in mouse uterus during the common pathway of parturition," American Journal of Obstetrics and Gynecology, vol. 198, no. 5, pp. 539.e1-539.e8, 2008.

[19] J. Folkman and M. Klagsbrun, "Angiogenic factors," Science, vol. 235, no. 4787, pp. 442-447, 1987.

[20] R. J. Torry and B. J. Rongish, "Angiogenesis in the uterus: potential regulation and relation to tumor angiogenesis," American Journal of Reproductive Immunology, vol. 27, no. 3-4, pp. 171-179, 1992.

[21] M. Zygmunt, F. Herr, K. Münstedt, U. Lang, and O. D. Liang, "Angiogenesis and vasculogenesis in pregnancy," European Journal of Obstetrics \& Gynecology and Reproductive Biology, vol. 110, pp. S10-S18, 2003.

[22] M. Jeansson, A. Gawlik, G. Anderson et al., "Angiopoietin-1 is essential in mouse vasculature during development and in response to injury," The Journal of Clinical Investigation, vol. 121, no. 6, pp. 2278-2289, 2011.

[23] G. D. Yancopoulos, S. Davis, N. W. Gale, J. S. Rudge, S. J. Wiegand, and J. Holash, "Vascular-specific growth factors and blood vessel formation," Nature, vol. 407, no. 6801, pp. 242-248, 2000.

[24] A. L. Conroy, E. I. Lafferty, F. E. Lovegrove et al., "Whole blood angiopoietin-1 and -2 levels discriminate cerebral and severe (non-cerebral) malaria from uncomplicated malaria," Malaria Journal, vol. 8, no. 1, p. 295, 2009.

[25] P. Kaufmann, S. Black, and B. Huppertz, "Endovascular trophoblast invasion: implications for the pathogenesis of intrauterine growth retardation and preeclampsia," Biology of Reproduction, vol. 69, no. 1, pp. 1-7, 2003.

[26] E. Leinonen, K.-A. Wathén, H. Alfthan et al., "Maternal serum angiopoietin-1 and -2 and tie-2 in early pregnancy ending in preeclampsia or intrauterine growth retardation," The Journal of Clinical Endocrinology \& Metabolism, vol. 95, no. 1, pp. 126-133, 2010.

[27] F. E. Lovegrove, N. Tangpukdee, R. O. Opoka et al., "Serum angiopoietin-1 and -2 levels discriminate cerebral malaria from uncomplicated malaria and predict clinical outcome in African children," PloS One, vol. 4, no. 3, p. e4912, 2009.

[28] L. K. Erdman, A. Dhabangi, C. Musoke et al., "Combinations of host biomarkers predict mortality among Ugandan children with severe malaria: a retrospective case-control study," PLoS One, vol. 6, no. 2, p. e17440, 2011.

[29] V. Jain, N. W. Lucchi, N. O. Wilson et al., "Plasma levels of angiopoietin-1 and -2 predict cerebral malaria outcome in Central India," Malaria Journal, vol. 10, no. 1, p. 383, 2011.

[30] A. J. Umbers, E. H. Aitken, and S. J. Rogerson, "Malaria in pregnancy: small babies, big problem," Trends in Parasitology, vol. 27, no. 4, pp. 168-175, 2011.

[31] P. Arbeille, G. Carles, M. Georgescu et al., "Consequences of reduced umbilical and increased foetal cerebral flow during malaria crisis on foetal behaviour," Parasitology, vol. 126, no. 6, pp. 513-519, 2003.

[32] E. K. Dorman, C. E. Shulman, J. Kingdom et al., "Impaired uteroplacental blood flow in pregnancies complicated by 
falciparum malaria," Ultrasound in Obstetrics and Gynecology, vol. 19, no. 2, pp. 165-170, 2002.

[33] K. L. Silver, A. L. Conroy, R. G. F. Leke et al., "Correction: circulating soluble endoglin levels in pregnant women in Cameroon and Malawi-associations with placental malaria and fetal growth restriction," PLoS One, vol. 6, no. 10, 2011.

[34] R. W. Steketee, M. E. Parise, B. L. Nahlen, and C. Menendez, "The burden of malaria in pregnancy in malaria-endemic areas," The American Journal of Tropical Medicine and Hygiene, vol. 64, 1_suppl, pp. 28-35, 2001.

[35] L. Apel-Sarid, A. Levy, G. Holcberg, and E. Sheiner, "Placental pathologies associated with intra-uterine fetal growth restriction complicated with and without oligohydramnios," Archives of Gynecology and Obstetrics, vol. 280, no. 4, pp. 549-552, 2009.

[36] D. Kappou, S. Sifakis, V. Androutsopoulos, A. Konstantinidou, D. A. Spandidos, and N. Papantoniou, "Placental mRNA expression of angiopoietins (Ang)-1, Ang-2 and their receptor Tie-2 is altered in pregnancies complicated by preeclampsia," Placenta, vol. 35, no. 9, pp. 718-723, 2014.

[37] F. Roviezzo, S. Tsigkos, A. Kotanidou et al., "Angiopoietin-2 causes inflammation in vivo by promoting vascular leakage," Journal of Pharmacology and Experimental Therapeutics, vol. 314, no. 2, pp. 738-744, 2005.

[38] S. Ong, G. Lash, and P. N. Baker, "Angiogenesis and placental growth in normal and compromised pregnancies," Best Practice \& Research Clinical Obstetrics \& Gynaecology, vol. 14, no. 6, pp. 969-980, 2000.

[39] A. L. Conroy, K. L. Silver, K. Zhong et al., "Complement activation and the resulting placental vascular insufficiency drives fetal growth restriction associated with placental malaria," Cell Host \& Microbe, vol. 13, no. 2, pp. 215-226, 2013. 


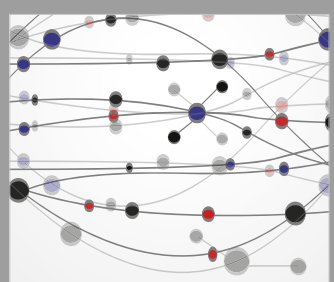

The Scientific World Journal
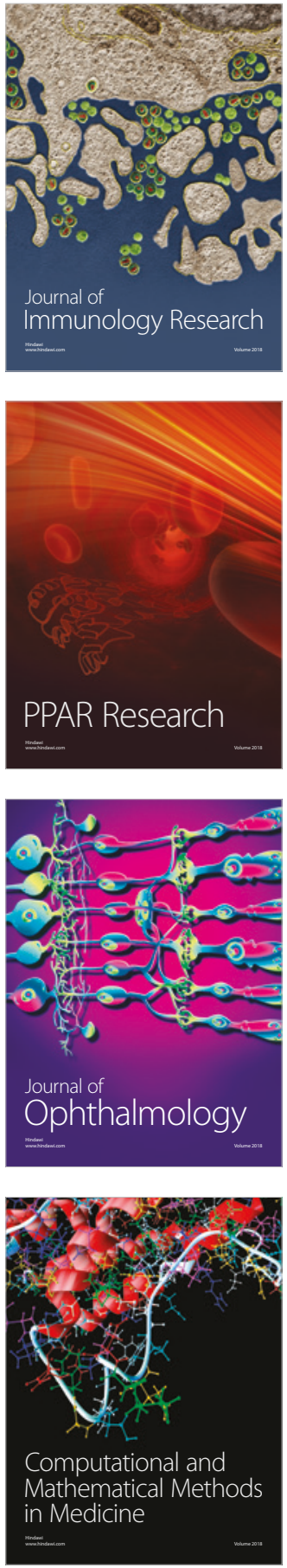

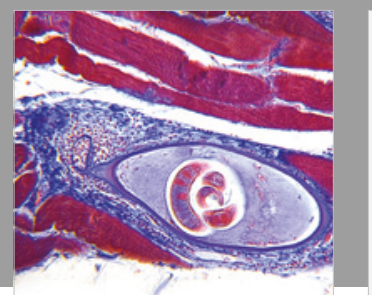

Gastroenterology Research and Practice

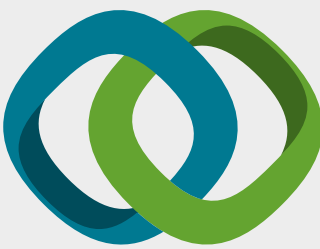

\section{Hindawi}

Submit your manuscripts at

www.hindawi.com
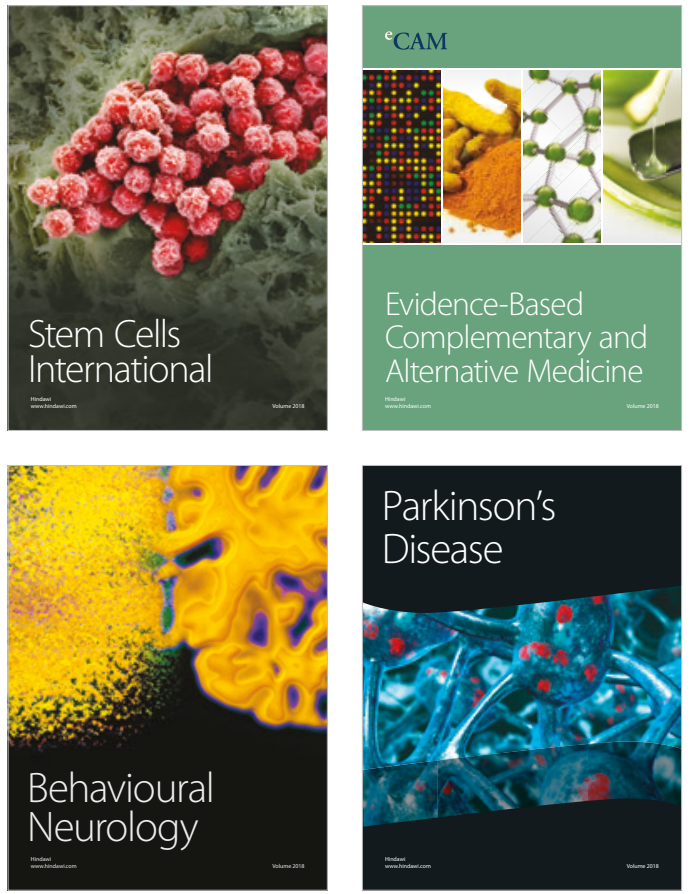

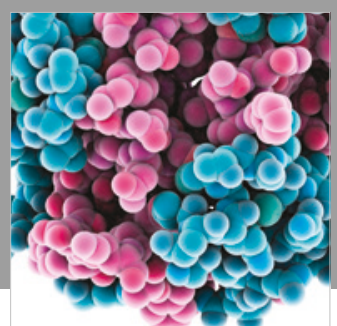

ournal of

Diabetes Research

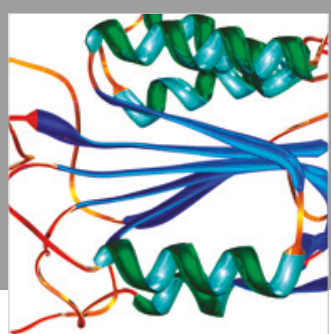

Disease Markers
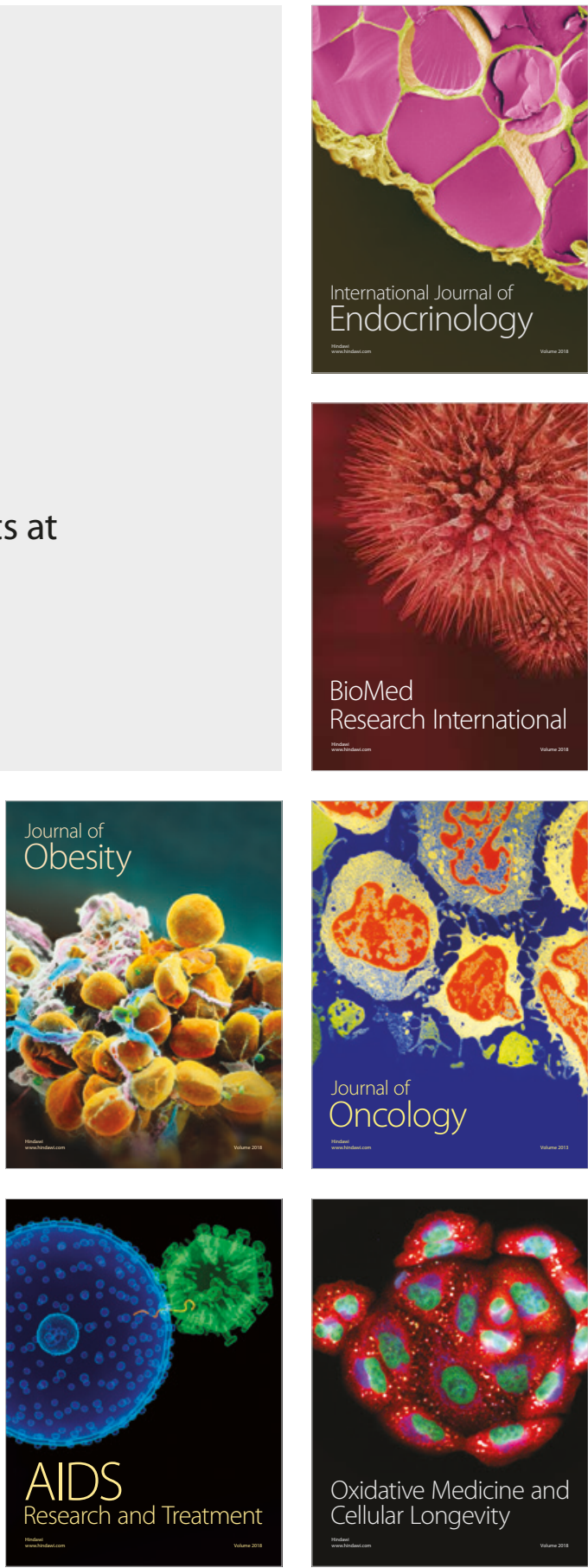